\title{
A MODEL FOR SIBLING EFFECTS IN MAN
}

\author{
LINDON EAVES \\ Department of Genetics, University of Birmingham, Birmingham B15 2TT
}

Received 7.x.75

\section{SUMmary}

\begin{abstract}
A model is developed to specify the environmental effect of one sibling on another for a polygenic trait. Such effects are detectable in principle and the approach is illustrated with twin data relating to psychoticism. The relationship between the model and those employed in the treatment of kin selection is indicated.
\end{abstract}

\section{INTRODUCTION}

Such data as are available suggest that much variation in human behavioural measurements is consistent with causation by the independent and additive effects of genes and environment. Obviously the resolution of many studies is poor, but even so data are not exploited to the full in testing the assumptions of the additivity and independence of genetical and environmental components.

Several kinds of effect can create non-independence of genes and environment, including cultural effects due to the influence of parent on offspring, but in this paper we consider the role of sibling effects in human variation and show how existing twin data might be examined afresh in order to assess the possible role of such effects in human populations.

The human family is such that one sibling constitutes part of the environment in which another develops. If the phenotype of one sibling influences the behaviour of another we may be said to have a "sibling effect". In so far as phenotypic variation is genetically determined the genes of an individual may exert an indirect influence on the phenotype of those in his family as well as having a direct contribution to the development of his own phenotype. Siblings, for example, may " compete " or " co-operate", depending on whether the presence in the family of a high-scoring sibling inhibits or facilitates the development of other siblings.

A consequence of the genetic determination of phenotypic variation may be that part of the environmental variation we observe is due to genetical heterogeneity of the individuals who influence the development of others. A further consequence, for individuals who are reared with relatives, may be that genetical and environmental effects covary. Recognising this possibility provides a basis for producing a model of sibling effects and designing studies to aid their detection, as we shall see from the following treatment.

\section{THE MODEL}

For simplicity we start by considering the effects of a single locus with two equally frequent alleles, $A, a$. Later, we shall consider the effects of loci for which the allele frequencies are unequal. For a population in Hardy- 
Weinberg equilibrium we expect the three genotypes at this locus to be represented in the frequencies $\frac{1}{4} A A: \frac{1}{2} A a: \frac{1}{4} a a$. We regard every allele, potentially, as having two effects when individuals are raised in families. It affects, directly, the phenotype of the individual who carries it, but it may also affect, indirectly, the phenotype of another individual who is reared with someone who carries the allele.

On a suitable scale we may represent the direct and indirect effects of the locus as follows:

$\begin{array}{llll}\text { Genotype } & A A & A a & a a \\ \text { Direct effect on phenotype } & +d_{a} & h_{a} & -d_{a} \\ \text { Indirect effect on sibling } & +d_{a}^{\prime} & h_{a}^{\prime} & -d_{a}^{\prime}\end{array}$

The "direct" and "indirect" effects need not have the same sign. When the direct and indirect effects have the same sign, i.e. when an allele increases the phenotypic expression of its carrier and of a sibling, we shall speak of "co-operation". When the effects have opposite sign, i.e. when an advantageous allele for the carrier creates a disadvantageous environment for a sibling, we shall refer to "competition ".

We shall consider only pairs of individuals reared together, although a similar treatment could be adopted with larger families. Suppose, for example, we have a pair of individuals of genotypes $A A$ and $A a$ at this locus. The contribution of the locus to the phenotypic scores of these individuals would be $+d_{a}+h_{a}^{\prime}$, and $h_{a}+d_{a}^{\prime}$ respectively. We may represent the phenotypic effects on all possible pairs in the same way. In writing our model this way we have assumed that the " direct " and " indirect" effects are additive, that is, there is no genotype-environment interaction. Four additional parameters would be required to specify such interactions fully in our model.

The frequencies with which each type of pair occur in the population will depend on the kind of relationship between the members of the pairs. In a population of $M Z$ twins, for example, we expect no pairs of the $A A, A a$ type. If we consider a population of randomly associated unrelated individuals we shall find such pairs account for a quarter of all possible pairs (when the $A$ and $a$ alleles are equally frequent).

In table 1 we give the frequencies of pairs of each type for three kinds of relationship, $M Z$ twins, $D Z$ twins (full siblings) and unrelated individuals reared in the same family. We give the expected frequencies given that the gene frequencies are equal and for the more general case in which we represent the frequencies of $A$ and $a$ by $u_{a}$ and $v_{a}$ respectively. We now ask "What is the contribution of the locus $A, a$ to the covariance between members of pairs and to the variation within pairs?" We can see that, since the gene frequencies are assumed to be the same in the three kinds of family, we expect the contribution of the direct effects $\left(d_{a}\right.$ and $\left.h_{a}\right)$ to the phenotypic variation expected to be the same for each group. Similarly, we expect the contribution of the "indirect" effects $\left(d_{a}^{\prime}\right.$ and $\left.h_{a}^{\prime}\right)$ to be the same. That is, we expect the genetic and environmental variance to be the same for each group. Inspection of table 1, however, shows that the direct and indirect effects are not necessarily independent. In $M Z$ twins, at one extreme, we find that the direct and indirect effects are completely correlated since every individual is reared in the presence of a sibling of identical genotype. At the other extreme, however, individuals who are fostered with 


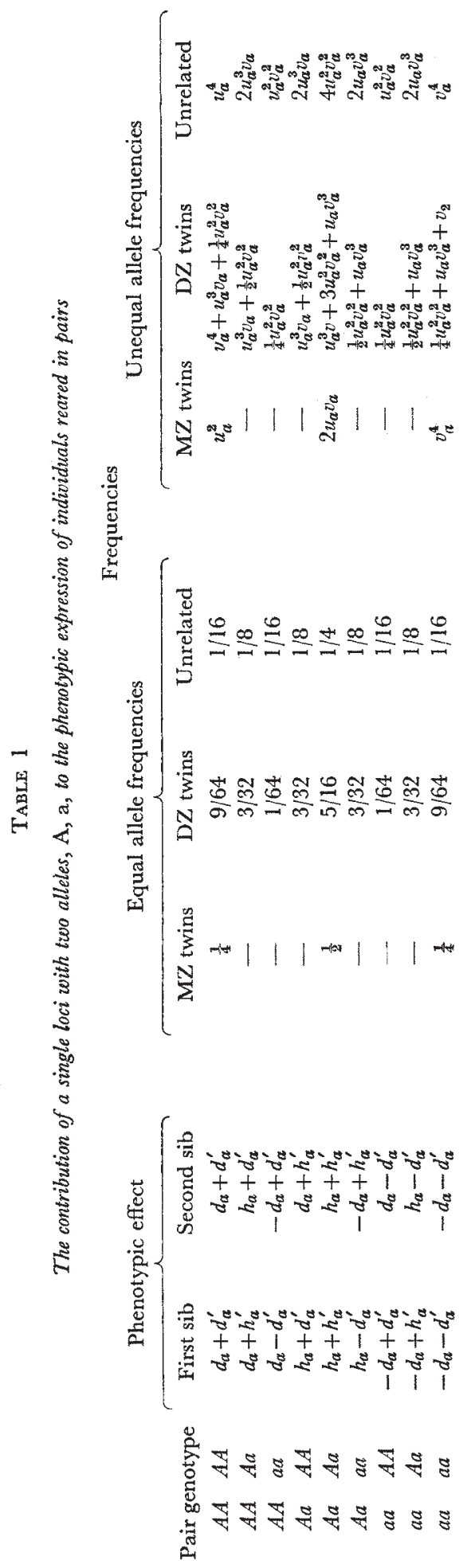


unrelated foster-siblings will receive environmental effects from their siblings which are assumed to be independent of their own genotypes. Thus, even though the allele frequencies are assumed to be constant over groups, the contribution to individual differences of covariation between the direct and indirect effects ("genotype-environmental covariance") is expected to decline with decreasing relationship between the individuals reared together. For this reason, we expect the effects of competition and co-operation to lead to heterogeneity of total variance between the three groups we have considered here.

We may quantify this intuitive argument by obtaining, algebraically, the contribution of the locus to the total variance of monozygotic and dizygotic twins, and of individuals reared with randomly chosen unrelated foster siblings. For the simple case of equal allele frequencies we take one sib of each pair (say the first) and multiply the square of its phenotypic effect by the frequency of such pairs in the population. Adding over all pairs and subtracting the square of the mean we get (for $M Z$ individuals)

$$
\begin{aligned}
\sigma_{A}^{2} & =\frac{1}{4}\left(d_{a}+d_{a}^{\prime}\right)^{2}+\frac{1}{2}\left(h_{a}+h_{a}^{\prime}\right)^{2}+\frac{1}{4}\left(-d_{a}-d_{a}^{\prime}\right)^{2}-\left(\frac{1}{2} h_{a}+\frac{1}{2} h_{a}^{\prime}\right)^{2} \\
& =\frac{1}{2}\left(d_{a}^{2}+2 d_{a} d_{a}^{\prime}+d_{a}^{\prime 2}\right)+\frac{1}{4}\left(h_{a}^{2}+2 h_{a} h_{a}^{\prime}+h_{a}^{\prime 2}\right) .
\end{aligned}
$$

We can easily extend this treatment to the cumulative effects of many independent loci simply by adding the contributions of the individual loci. This will be appropriate providing the loci are in linkage equilibrium and there is no epistasis. This gives, for

where

$$
\sigma_{M Z}^{2}=\frac{1}{2} D+D^{\prime}+\frac{1}{2} D^{\prime \prime}+\frac{1}{4} H+\frac{1}{2} H^{\prime}+\frac{1}{4} H^{\prime \prime},
$$

$$
D=\Sigma_{a} d_{a}^{2}, D^{\prime}=\Sigma_{a} d_{a} d_{a}^{\prime}, D^{\prime \prime}=\Sigma_{a} d_{a}^{\prime 2}, H=\Sigma_{a} h_{a}^{2}, H^{\prime}=\Sigma_{a} h_{a} h_{a}^{\prime}
$$

and

$$
H^{\prime \prime}=\Sigma_{a} h_{a}^{\prime 2} .
$$

Similary, the total variance of dizygotic twins is found to be:

$$
\sigma_{D Z}^{2}=\frac{1}{2} D+\frac{1}{2} D^{\prime}+\frac{1}{2} D^{\prime \prime}+\frac{1}{4} H+\frac{1}{8} H^{\prime}+\frac{1}{4} H^{\prime \prime}
$$

and the total variance of individuals reared with foster siblings is

$$
\sigma_{U}^{2}=\frac{1}{2} D+\frac{1}{2} D^{\prime \prime}+\frac{1}{4} H+\frac{1}{4} H^{\prime \prime}
$$

Thus, in the presence of co-operation ( $D^{\prime}$ and $H^{\prime}$ being positive) we expect

$$
\sigma_{M Z}^{2}>\sigma_{D Z}^{2}>\sigma_{U}^{2}
$$

This provides a fairly powerful test of co-operative effects which would normally be obscured by any more traditional analysis of intra-class correlations. If $D^{\prime}$ and $H^{\prime}$ are negative, however, i.e. if there is competition between siblings, we expect

$$
\sigma_{M Z}^{2}<\sigma_{D Z}^{2}<\sigma_{U}^{2}
$$

Even if we lack the data on unrelated individuals reared together we would be led to suspect competition or co-operation as a basis of individual differences if we found that the variances for monozygotic and dizygotic twins were 
different. In writing our expectations we have ignored the contribution of additional environmental factors such as those due to cultural differences and environmental effects specific to particular individuals.

Our expectations for the total variances, however, do not exhaust all the information contained in a sample of relatives. For each group we may obtain the covariance between members of the sibling pairs and the variance within families.

For $M Z$ twins we have; quite simply:

$$
W_{M Z}=\frac{1}{2} D+D^{\prime}+\frac{1}{2} D^{\prime \prime}+\frac{1}{4} H+\frac{1}{2} H^{\prime}+\frac{1}{4} H^{\prime \prime},
$$

there being no contribution to the variance within pairs $\left(\sigma_{W M Z}^{2}\right)$ except for that of random environmental factors.

For $D Z$ twins and full siblings we have, for the contribution of locus $A, a$,

$$
\begin{aligned}
W_{D Z}=\frac{9}{64}\left(d_{a}-d_{a}^{\prime}\right)\left(d_{a}+d_{a}^{\prime}\right) & +\frac{3}{32}\left(d_{a}+h_{a}^{\prime}\right)\left(h_{a}+d_{a}^{\prime}\right)+\ldots \\
& +\frac{9}{64}\left(-d_{a}-d_{a}^{\prime}\right)\left(-d_{a}-d_{a}^{\prime}\right)-\left(\frac{1}{2} h_{a}+\frac{1}{2} h_{a}^{\prime}\right)\left(\frac{1}{2} h_{a}+\frac{1}{2} h_{a}^{\prime}\right) .
\end{aligned}
$$

Summing over many loci we get

$$
W_{D Z}=\frac{1}{4} D+D^{\prime}+\frac{1}{4} D^{\prime \prime}+\frac{1}{16} H+\frac{1}{2} H^{\prime}+\frac{1}{16} H^{\prime \prime},
$$

i.e. although the contribution of $D, D^{\prime \prime}, H$ and $H^{\prime \prime}$ to the covariance is reduced by reducing the degree of relationship between members of a pair we find that the contribution of $D^{\prime}$ and $H^{\prime}$ persists undiminished. An important consequence of this fact is that the existence of competition may result in negative covariances (and hence correlations) between siblings because the contribution of large negative $D^{\prime}$ and $H^{\prime}$ terms outweigh the positive contribution of the other components which have relatively small coefficients. Even if the covariances are not negative, the presence of competition would account for sibling covariances which are too small to be explained by any simple genetical mechanism.

The effect on the covariance of pairs is even more marked in the expectation for the covariance of unrelated individuals reared together which is simply

$$
W_{U}=D^{\prime}+\frac{1}{2} H^{\prime},
$$

i.e. the covariance between unrelated individuals reared together contains nothing of $D, D^{\prime \prime}, H$ and $H^{\prime \prime}$, but has a full measure of the competitive and co-operative effects. Individuals of this kind are likely to be the most use for the detection of competition since, in the absence of cultural effects which would inflate $W_{U}$, there is no genetical similarity between individuals to mask any effect of competition.

We notice that even though $W_{D Z}$ and $W_{U}$ both contain $D^{\prime}+\frac{1}{2} H^{\prime}$ the total variances contain a smaller component of $D^{\prime}$ and $H^{\prime}$. This is because the competition/co-operation parameters make a contribution to the variances within families which is opposite in sign to their contribution to the covariances between individuals in families. Thus, co-operation increases the similarity between siblings by reducing the variance within families as much as by increasing the variance between families.

The simplest way of obtaining the expectation for the variance within pairs is to multiply half of the square of each intrapair difference by its 
expected frequency in the population, adding the result for all kinds of pair. So, for the variance within pairs of natural siblings (or $D Z$ twins) we have:

$$
\begin{aligned}
\sigma_{W D Z}^{2}=\frac{9}{64}\left(d_{a}+d_{a}^{\prime}-d_{a}-d_{a}^{\prime}\right)^{2} & +\frac{3}{32}\left(d_{a}+h_{a}^{\prime}-h_{a}-d_{a}^{\prime}\right)^{2} \ldots \\
& +\frac{3}{32}\left(-d_{a}+h_{a}^{\prime}-h_{a}+d_{a}^{\prime}\right)^{2}+\frac{9}{64}\left(-d_{a}-d_{a}^{\prime}+d_{a}+d_{a}^{\prime}\right)^{2} .
\end{aligned}
$$

Adding over the contribution of many independent loci we obtain:

Similarly,

$$
\sigma_{W D Z}^{2}=\frac{1}{4} D-\frac{1}{2} D^{\prime}+\frac{1}{4} D^{\prime \prime}+\frac{3}{16} H-\frac{3}{8} H^{\prime}+\frac{3}{16} H^{\prime \prime} .
$$

$$
\sigma_{W U}^{2}=\frac{1}{2} D-D^{\prime}+\frac{1}{2} D^{\prime \prime}+\frac{1}{4} H-\frac{1}{2} H^{\prime}+\frac{1}{4} H^{\prime \prime} .
$$

So far, we have assumed that the allele frequencies are equal at all the loci involved in the expression of the trait. Clearly this is unrealistic. We may, however, generalise our expectations to the case of unequal gene frequencies.

We put

$$
\begin{aligned}
& D_{R}=\Sigma_{a} 4 u_{a} v_{a}\left[d_{a}+\left(v_{a}-u_{a}\right) h_{a}\right]^{2} \\
& D_{R}^{\prime}=\Sigma_{a} 4 u_{a} v_{a}\left[d_{a}+\left(v_{a}-u_{a}\right) h_{a}\right]\left[d_{a}^{\prime}+\left(v_{a}-u_{a}\right) h_{a}^{\prime}\right] \\
& D_{R}^{\prime \prime}=\Sigma_{a} 4 u_{a} v_{a}\left[d_{a}^{\prime}+\left(v_{a}-u_{a}\right) \dot{h}_{a}^{\prime}\right]^{2} \\
& H_{R}=\Sigma_{a} 16 u_{a}^{2} v_{a}^{2} h_{a}^{2} \\
& H_{R}^{\prime}=\Sigma_{a} 16 u_{a}^{2} v_{a}^{2} h_{a} h_{a}^{\prime} \\
& H_{R}^{\prime \prime}=\Sigma_{a} 16 u_{a}^{2} v_{a}^{2} h_{a}^{\prime 2}
\end{aligned}
$$

and substitute $D_{R}, D_{R}^{\prime}$ etc. for $D, D^{\prime}$ etc. in the expectations for the variances and covariances above. We see that when $u_{a}=v_{a}=\frac{1}{2}$ to every locus $D_{R}$ simplifies to $D, H_{R}$ to $H$ etc. (see Mather and Jinks, 1971).

\section{Example}

The approach can be illustrated by an analysis of twin data for psychoticism. We consider the psychoticism scores of pairs of male twins derived for their responses to the PEN questionnaire.

The raw data were subjected to a square-root transformation to secure a scale on which the contribution of genes and environment were additive, as far as could be determined (see Eaves and Eysenck, 1976).

The mean squares within and between families were computed for 120 pairs of $M Z$ and 59 pairs of $D Z$ twins on the transformed scale of measurement. These are given in table 2. Eaves and Eysenck (1976) found that these data were barely consistent with a simple genetical model which assumed additive gene action and environmental variation within families and the anomalies in the data are such as to preclude any interpretation in terms of cultural effects or genetical non-additivity. We see, however, that the mean-squares follow a pattern strongly suggestive of competition since the total variance for $D Z$ twins is somewhat (though not quite significantly) larger than that for $M Z$ twins, and the mean square between $D Z$ pairs is actually less than that within $D Z$ pairs. We show how both these 
anomalies can be explained in terms of a simplified co-operation-competition model. We assume:

1. All genetical effects, direct or indirect, are additive (i.e. we omit $H_{R}$, and $H_{R}^{\prime} H_{R}^{\prime \prime}$ from our expectations).

2. Mating is random, so gene effects are independent.

3. All environmental effects which do not result from sibling effects contribute to variation within families (i.e. there are no $E_{2}$ effects).

We can represent our assumptions in terms of the model given in table 2. We obtain the expectations of mean-squares by recognising that the mean

TABLE 2

Mean squares and their expectations in terms of a simplified sibling effects model for male psychoticism data

\begin{tabular}{|c|c|c|c|c|c|c|}
\hline \multirow[b]{2}{*}{ Twin type } & \multirow[b]{2}{*}{ Item } & \multirow[b]{2}{*}{ d.f. } & \multirow[b]{2}{*}{ Mean square } & \multicolumn{3}{|c|}{ Expected contribution of $\dagger$} \\
\hline & & & & $\left(D_{R}+D_{R}^{\prime \prime}\right)$ & $D_{R}^{\prime}$ & $E_{1}$ \\
\hline \multirow[t]{2}{*}{ Monozygotic } & Between pairs & 119 & $0 \cdot 0206$ & 1 & 2 & 1 \\
\hline & Within pairs & 120 & $0 \cdot 0089$ & - & - & 1 \\
\hline \multirow[t]{2}{*}{ Dizygotic } & Between pairs & 58 & $0 \cdot 0176$ & $\frac{3}{4}$ & $1 \frac{1}{2}$ & 1 \\
\hline & Within pairs & 59 & 0.0195 & $\frac{1}{4}$ & $-\frac{1}{2}$ & 1 \\
\hline
\end{tabular}

$\dagger$ See text for definition and explanation of terms.

square between pairs comprises the corresponding within-pair variance component plus twice the appropriate between-family component. We have already obtained the expectations of $\sigma_{W M Z}^{2}$ and $\sigma_{W D Z}^{2}$. The expectations of the corresponding between-family variance components are equal to those for the covariances, $W_{M Z}$ and $W_{D Z}$ respectively.

We see that $D_{R}$ and $D_{R}^{\prime \prime}$ have identical coefficients in the expectations of all the statistics so they can never be separated in these data (or in any other data in which individuals are reared in pairs). We can, however, estimate $\left(D_{R}+D_{R}^{\prime \prime}\right), D_{R}^{\prime}$ and $E_{1}$ by weighted least squares leaving us one d.f. for testing our model. Our estimates are

$$
\begin{array}{rr}
\left(D_{R} \hat{+} D_{R}^{\prime \prime}\right)= & 0.02712 \pm 0.00785 \\
\hat{D}_{R}^{\prime}= & -0.00773 \pm 0.00373 \\
\hat{E}_{1}= & 0.00889 \pm 0.00114 .
\end{array}
$$

The weighted sum of squared residuals is 0.004 which is, approximately, distributed as $\chi_{1}^{2}$. The probability of residuals as large as, or larger than these is thus 0.98 . The model fits exceptionally well. This probably reflects the element of post-hoc rationalisation applied here to a particular set of anomalous data. We should not expect such a close fit in a repeated study. The estimates we obtain, however, do illustrate the principle of competition very clearly. In addition to our significant $\left(D_{R} \hat{+} D_{R}^{\prime \prime}\right)$ we have a significant negative estimate of $D_{R}^{\prime}$ (i.e. negative genotype-environmental covariance) implying that the genes which control the development of normal behaviour do so at the expense of other individuals reared in the same family, since relatively normal behaviour in one sibling tends to be accompanied by relative abnormality in another, and vice versa. 
In the presence of sibling effects there is no simple " heritability" which can be applied to the population as a whole since the "environmental" effects in $D_{R}^{\prime \prime}$ are confounded with the genetical effects included in $D_{R}$, and the contribution of genotype-environmental covariation, $D_{R}^{\prime}$, depends on the degree of relationship between the members of a pair. We can, however,

TABLE 3

The specification of sibling effects for a polygenic trait $\dagger$ when mating is random

\begin{tabular}{|c|c|c|c|c|}
\hline Relationship & & $\begin{array}{l}\text { Genetical } \\
\text { variance }\end{array}$ & $\begin{array}{c}\text { Environmental } \\
\text { variance }\end{array}$ & $\begin{array}{l}\text { Genotype-environmental } \\
\text { covariance }\end{array}$ \\
\hline $\begin{array}{l}\text { Monozygotic twins } \\
\text { reared together }\end{array}$ & $\begin{array}{l}\text { Covariance } \\
\text { Within-pair variance } \\
\text { Total variance }\end{array}$ & $\begin{array}{c}\frac{1}{2} D_{R}+\frac{1}{4} H_{R} \\
- \\
\frac{1}{2} D_{R}+\frac{1}{4} H_{R}\end{array}$ & $\begin{array}{l}+\frac{1}{2} D_{R}^{\prime \prime}+\frac{1}{4} H_{R}^{\prime \prime} \\
+\frac{1}{2} D_{R}^{\prime \prime}+\frac{1}{4} H_{R}^{\prime \prime}\end{array}$ & $\begin{array}{l}+D_{R}^{\prime}+\frac{1}{2} H_{\Omega}^{\prime} \\
+D_{R}^{\prime}+\frac{1}{2} H_{R}^{\prime}\end{array}$ \\
\hline $\begin{array}{l}\text { Dizygotic twins } \\
\text { reared together }\end{array}$ & $\begin{array}{l}\text { Covariance } \\
\text { Within-pair variance } \\
\text { Total variance }\end{array}$ & $\begin{array}{l}\frac{1}{4} D_{R}+\frac{1}{16} H_{R} \\
\frac{1}{4} D_{R}+\frac{3}{16} H_{R} \\
\frac{1}{2} D_{R}+\frac{1}{4} H_{R}\end{array}$ & $\begin{array}{l}+\frac{1}{4} D_{R}^{\prime \prime}+\frac{1}{16} H_{R}^{\prime \prime} \\
+\frac{1}{4} D_{R}^{\prime \prime}+\frac{3}{16} H_{R}^{\prime \prime} \\
+\frac{1}{2} D_{R}^{\prime \prime}+\frac{1}{4} H_{R}^{\prime \prime}\end{array}$ & $\begin{array}{l}+D_{R}^{\prime}+\frac{1}{2} H_{R}^{\prime} \\
-\frac{1}{2} D_{R}^{\prime}-\frac{3}{8} H_{R}^{\prime} \\
+\frac{1}{2} D_{R}^{\prime}+\frac{1}{8} H_{R}^{\prime}\end{array}$ \\
\hline $\begin{array}{l}\text { Unrelated individuals } \\
\text { reared together }\end{array}$ & $\begin{array}{l}\text { Covariance } \\
\text { Within-pair variance } \\
\text { Total variance }\end{array}$ & $\begin{array}{c}- \\
\frac{1}{2} D_{R}+\frac{1}{4} H_{R} \\
\frac{1}{2} D_{R}+\frac{1}{4} H_{R}\end{array}$ & $\begin{array}{c}- \\
+\frac{1}{2} D_{R}^{\prime \prime}+\frac{1}{4} H_{R}^{\prime \prime} \\
+\frac{1}{2} D_{R}^{\prime \prime}+\frac{1}{4} H_{R}^{\prime \prime}\end{array}$ & $\begin{array}{r}D_{R}^{\prime}+\frac{1}{2} H_{R}^{\prime} \\
-D_{R}^{\prime}-\frac{1}{2} H_{R}^{\prime} \\
\end{array}$ \\
\hline Singletons & Total variance & $\frac{1}{2} D_{R}+\frac{1}{4} H_{R}$ & 一 & - \\
\hline
\end{tabular}

$\dagger$ We have not added the contribution of environmental variation due to other factors.

express the variation which depends ultimately on genetical polymorphism (whether due to " direct" or "indirect" effects or to their covariation) as a proportion of the total variance for each kind of relationship. Thus, for psychoticism in males, as we have measured it, we find a model with only additive genetical effects $\left(D_{R}, D_{R}^{\prime}\right.$ and $\left.D_{R}^{\prime \prime}\right)$ and specific environmental effects $\left(E_{1}\right)$ is consistent with the data. We can, therefore, write for $M Z$ twins:

$$
\begin{aligned}
h_{M Z}^{2} & =\frac{\frac{1}{2}\left(\widehat{D}_{R}+2 \widehat{D}_{R}^{\prime}+\hat{D}_{R}^{\prime \prime}\right)}{\frac{1}{2}\left(\widehat{D}_{R}+2 \widehat{D}_{R}^{\prime}+\widehat{D}_{R}^{\prime \prime}\right)+\widehat{E}_{1}} \\
& =0.397
\end{aligned}
$$

and

$$
\begin{aligned}
h_{D Z}^{2} & =\frac{\frac{1}{2}\left(\widehat{D}_{R}+\widehat{D}_{R}^{\prime}+\widehat{D}_{R}^{\prime \prime}\right)}{\frac{1}{2}\left(\widehat{D}_{R}+\widehat{D}_{R}^{\prime}+\widehat{D}_{R}^{\prime \prime}\right)+\hat{E}_{1}} \\
& =0.522 .
\end{aligned}
$$

Our model, if it is generally satisfactory, should lead us to predict the variance and covariance of unrelated individuals reared together. We expect the total variance of unrelated individuals reared together to be $\frac{1}{2}\left(D_{R} \hat{+} D_{R}^{\prime \prime}\right)+\hat{E}_{1}$, and the covariance to be $\hat{D}_{R}^{\prime}$.

The correlation for unrelated individuals reared together should thus be about $-0 \cdot 30$, and we would expect the proportion of the total variation ultimately attributable to genetical factors to be 


$$
\begin{aligned}
h_{U T}^{2} & =\frac{\frac{1}{2}\left(D_{R} \hat{+} D_{R}^{\prime \prime}\right)}{\frac{1}{2}\left(D_{R} \hat{+} D_{R}^{\prime \prime}\right)+\hat{E}_{1}} \\
& =0.604 .
\end{aligned}
$$

From the three ratios we can see that competition serves to dampen the effect of genetical variation in familes of related individuals. The apparent genetical variation will be less in families of most closely related individuals. The reverse effect would be apparent if the sibling effects were co-operative rather than competitive.

There are, of course, other situations which might be exploited in testing for the effects of competition and co-operation. In the absence of genotypeenvironmental interaction, for example, we might expect singletons to differ in variance from individuals reared in pairs because their variance is not expected to contain any $D_{R}^{\prime}, D_{R}^{\prime \prime}, H_{R}^{\prime}$ and $H_{R}^{\prime \prime}$. If $D_{R}^{\prime}$ and $H_{R}^{\prime}$ were zero, that is if the genes showing direct effects did not also show indirect "sibling" effects and vice versa, we would expect singletons to be less variable than siblings because singletons lacked the additional source of environmental variability which comes from being exposed to the influence of a sibling. We thus expect the variance of singletons to be $\frac{1}{2} D_{R}+\frac{1}{4} H_{R}$, ignoring any additional variation due to non-sibling environmental effects.

\section{Discussion}

In table 3 we summarise the contribution of sibling effects to various statistics. Data on monozygotic and dizygotic twins reared together with the addition, if possible, of unrelated individuals reared together can provide the material for testing for sibling competition and co-operation. The most powerful single test would be that provided by the expected ranking of total variances for the different groups. The information necessary for such a test of competition or co-operation is lost by those studies of twins which concentrate on the analysis of correlations. The covariances between natural and foster siblings reared together, and the within-pair variances, provide further information which would be a guide to the likely magnitude of any competitive or co-operative effect. In particular, a negative covariance between $D Z$ twins and/or unrelated individuals reared together could suggest competition is contributing to variation for a particular trait. A close examination of the data underlying published twin correlations could reveal anomalies of the kind which would suggest the importance of competition or co-operation in the development of behaviour.

The biological significance of this, and similar models is obvious. Several workers have studied the evolutionary consequences of selection based on kinships rather than individuals (e.g. Hamilton, 1964, Maynard-Smith, 1964). A prerequisite for kin selection is that individuals are affected environmentally by the genetical variation of their relatives. If we detect substantial sibling effects for a partly inherited trait we have demonstrated the existence of the raw material for kin selection. If, in human populations, selection is acting on families rather than individuals we might expect traits related strongly to fitness to show the pattern of variation associated with sibling effects. On the other hand, if natural selection in man is chiefly operating at the individual level we would expect traits related to fitness to 
show little or no effect of the kind we have considered here. The approach presented here shows how sibling effects might be detected in practice and so yield some further insight into the evolutionary significance of the human family.

Acknowledgment.-This work is part of a Research Programme in psychogenetics supported by the MRC. The data chosen to illustrate the paper have been produced in collaboration with the Institute of Psychiatry, London University. I thank Professor J. L. Jinks for encouragement, Mrs K. Last and Mr N. G. Martin for discussion and many colleagues for their helpful criticism.

\section{REFERENCES}

EAVES, L. J., AND EYSENCK, H. J. 1976. A genotype-environmental model for psychoticism. For: Psychological Bulletin.

mamilton, w. D. 1964. The genetical evolution of social behavior. I. Fournal of theoretical Biology, 7, 1-16.

MATHER, K., AND JINKS, J. L. 1971. Biometrical genetics: the study of continuous variation. Chapman and Hall, London.

MAYNARD-SMith, J. 1964. Group selection and kin selection. Nature, 201, 1145-1147. 